\title{
Parameter counting in models with global symmetries
}

\author{
Joshua Berger* and Yuval Grossmant \\ Institute for High Energy Phenomenology \\ Newman Laboratory of Elementary Particle Physics \\ Cornell University, Ithaca, NY 14853, USA
}

\begin{abstract}
We present rules for determining the number of physical parameters in models with exact flavor symmetries. In such models the total number of parameters (physical and unphysical) needed to described a matrix is less than in a model without the symmetries. Several toy examples are studied in order to demonstrate the rules. The use of global symmetries in studying the minimally supersymmetric standard model (MSSM) is examined.
\end{abstract}

*Electronic address: jb454@cornell.edu

$\dagger$ Electronic address: yuvalg@lepp.cornell.edu 


\section{INTRODUCTION}

When modeling a physical system, it is important to understand the relationship between the symmetries in the model and the number of physical parameters involved. Consider for example a hydrogen atom in a uniform magnetic field. Before turning on the magnetic field, the hydrogen atom is invariant under spatial rotations, which are described by the $S O(3)$ group. Furthermore, there is an energy eigenvalue degeneracy of the Hamiltonian: states with different angular momenta have the same energy. This degeneracy is a consequence of the symmetry of the system.

When magnetic field is added to the system, it is conventional to pick a direction for the magnetic field without a loss of generality. Usually, we define the positive $z$ direction to be the direction of the magnetic field. Consider this choice more carefully. A generic uniform magnetic field would be described by three real numbers: the three components of the magnetic field. However, the magnetic field breaks the $S O(3)$ symmetry of the hydrogen atom system down to an $S O(2)$ symmetry of rotations in the plane perpendicular to the magnetic field. The one generator of the $S O(2)$ symmetry is the only valid symmetry generator now; the remaining two $S O(3)$ generators in the orthogonal planes are broken. These broken symmetry generators allow us to rotate the system such that the magnetic field points in the $z$ direction:

$$
O_{x z} O_{y z}\left(\begin{array}{c}
B_{x} \\
B_{y} \\
B_{z}
\end{array}\right)=\left(\begin{array}{c}
0 \\
0 \\
B_{z}^{\prime}
\end{array}\right),
$$

where $O_{x z}$ and $O_{y z}$ are rotations in the $x z$ and $y z$ planes respectively. The two broken generators were used to rotate away two unphysical parameters, leaving us with one physical parameter, the value of the magnetic field. That is, all measurable quantities in the system depend only on one new parameter, rather than the naïve three. In addition, the broken symmetry lifts the degeneracy of the energy eigenvalues.

The results described above are more generally applicable. Particularly, they are useful in studying the flavor physics of quantum field theories. Consider a gauge theory with matter content. This theory always has kinetic and gauge terms, which have a certain global symmetry $G_{f}$ on their own. However, in adding a potential, which consists of a linear combination of all renormalizable operators that respect the imposed symmetries, the global symmetry may be broken down to a smaller symmetry group. In breaking the symmetry, there is an added freedom to rotate away unphysical parameters, as when a magnetic field is added to the hydrogen atom system. In order to analyze this process, we define a few quantities. The added potential has coefficients that can be described by $N_{\text {general }}$ parameters 
in a general basis. The global symmetry $H_{f}$ of the entire model has fewer generators than $G_{f}$ and we call the difference in the number of generators $N_{\text {broken }}$. Finally, the quantity that we would ultimately like to determine is the number of parameters affecting physical measurements, $N_{\text {phys }}$. These numbers are related by the well-known rule [1] (for a review see, for example, Ref. [2])

$$
N_{\text {phys }}=N_{\text {general }}-N_{\text {broken }}
$$

Furthermore, this rule applies separately for both real parameters (masses and mixing angles) and phases. A general, $n \times n$ complex matrix can be parametrized by $n^{2}$ real parameters and $n^{2}$ phases. Imposing restrictions like Hermiticity or unitarity reduces the number of parameters required to describe the matrix. A Hermitian matrix can be described by $n(n+$ 1)/2 real parameters and $n(n-1) / 2$ phases, while a unitary matrix can be described by $n(n-1) / 2$ real parameters and $n(n+1) / 2$ phases.

The rule given by (2) can be applied to the standard model. We consider only terms involving fermions, stating results for the Higgs field when they are relevant. The Yukawa potential for the interactions in terms of the quark $S U(2)_{L}$ doublet, $Q_{L}$, the lepton $S U(2)$ doublet, $L_{L}$, the $S U(2)_{L}$ singlet fields, $U_{R}, D_{R}, E_{R}$, and the Higgs doublet, $H$, is

$$
V=Y_{i j}^{U}\left(\overline{Q_{L}}\right)_{i}\left(U_{R}\right)_{j} H+Y_{i j}^{D}\left(\overline{Q_{L}}\right)_{i}\left(D_{R}\right)_{j} \tilde{H}+Y_{i j}^{E}\left(\overline{L_{L}}\right)_{i}\left(E_{R}\right)_{j} \tilde{H}+\text { h.c. },
$$

where $Y^{F}$ are $3 \times 3$ complex matrices in a general basis. We use $\tilde{H}=\epsilon H^{*}$, where $\epsilon$ is the anti-symmetric matrix in $S U(2)_{L}$ space.

The interactions in this sector are parametrized by three complex $3 \times 3$ matrices, which contain a total of 54 parameters (27 real parameters and 27 phases) in a general basis. These parameters also break a large global symmetry of the kinetic and gauge terms in the model down to the familiar baryon number and lepton family number symmetries of the full standard model,

$$
U(3)_{Q} \times U(3)_{U} \times U(3)_{D} \times U(3)_{L} \times U(3)_{E} \rightarrow U(1)_{B} \times U(1)_{e} \times U(1)_{\mu} \times U(1)_{\tau} .
$$

While $U(3)^{5}$ has 45 generators, the remaining symmetry group has only 4 and thus $N_{\text {broken }}=$ 41. This broken symmetry allows us to rotate away a large number of the parameters by moving to a more convenient basis. Using (2), the number of physical parameters should be given by

$$
N_{\text {phys }}=54-41=13 .
$$

In addition, there are the three gauge couplings, the two Higgs parameters and the strong $\mathrm{CP}$ phase for a total of 19 parameters in the standard model. These parameters can be split into real parameters and phases. The five unitary matrices generating the symmetry of the 
kinetic and gauge terms have a total of 15 real parameters and 30 phases and the symmetry is broken down to a symmetry with only four phase generators. Thus,

$$
N_{\text {phys }}^{(r)}=27-15=12, \quad N_{\text {phys }}^{(i)}=27-26=1 .
$$

We interpret this result by saying that of the 12 real parameters, 9 are the fermion masses and three are the CKM matrix mixing angles. The one phase is the CP-violating phase of the CKM mixing matrix.

In studying new models, it is particularly important to properly count the number of parameters. The number of physical parameters is, in principle, the number of measurements required in order to fully determine a model. Once these measurements are made, it should be possible to test the model with all further measurements. The standard model is so successful because all the parameters have been measured to some extent and further measurements have verified significant predictions of the model to high precision. The current parametrization appears to be sufficient to describe the quark sector at scales below $100 \mathrm{GeV}[3]$. The failure of the SM parametrization in the lepton sector have been used as indicators of new lepton flavor physics [3].

In this paper, we extend the rule for parameter counting to theories where global symmetries are imposed on the potential terms. In particular, we consider cases where part of the flavor symmetry present in the kinetic and gauge terms is restored. In section 2, a rule for analyzing these cases is presented. Simple toy examples are discussed to highlight the use of the rule. In section 3, the rule is applied to studying global symmetry constraints in the MSSM. The results of imposing symmetries are compared to the constrained MSSM (cMSSM).

\section{RULES FOR PARAMETER COUNTING}

In general, we distinguish between two ways in which one could impose a global symmetry. The symmetry can be imposed on the whole model, or only on a specific sector. Clearly, a symmetry of a specific sector is broken by higher order terms. Yet, in terms of parameter counting we care about the tree level parameters. For example, in the SM the custodial symmetry is respected only by the Higgs sector and it is broken at one loop.

In the following, we study both cases and show that the general result is the same: the total number of parameters, $N_{\text {total }}$, needed to describe a model in a general basis is reduced compared to a model without such symmetries. The specific number of parameters needed in each case is different.

The most general type of terms on which we consider imposing a global symmetry has 
the form

$$
Y_{i j} \phi_{i}^{(1)} \phi_{j}^{(2)} \ldots
$$

where $\phi^{(1)}$ and $\phi^{(2)}$ have $n$ generations each, $Y$ is an $n \times n$ mixing matrix and ... represents other (flavor-singlet) factors that ensure that the term is a gauge group singlet. Multiple terms of the form (7) may be present. It is therefore possible that some of the symmetries imposed could hold for some terms, but be broken explicitly by others. Furthermore, if one or more of the gauge symmetries of the model is broken, then it is possible to allow the imposed symmetries to be broken by the gauge sector.

We start by looking at a simple toy model. Consider the leptonic sector of the standard model, but with an imposed $S U(2)$ symmetry such that two of the lepton masses are the same. Since the leptonic Yukawa matrix can be diagonalized without breaking any gauge symmetry, if the symmetry is imposed on the Yukawa sector, it will hold for the entire model. Thus, the cases of imposing the symmetry on the model and on the Yukawa sector only are the same for this choice of matter content. The only interaction term that it is necessary to consider for now is the third term in (3),$Y_{i j}^{E}\left(\overline{L_{L}}\right)_{i}\left(E_{R}\right)_{j} \tilde{H}$. As we show below, the result is that the total number of parameters required to describe this term in an arbitrary basis, $N_{\text {total }}$, is reduced from 18 to 15 .

In an arbitrary basis, we begin to decompose the matrix $Y^{E}$, first performing a polar decomposition [4]:

$$
Y^{E}=R \Phi
$$

where $R$ is Hermitian with positive eigenvalues and $\Phi$ is unitary. The next step is to perform a spectral decomposition on $R$ :

$$
Y^{E}=U^{\dagger} D U \Phi
$$

where $U$ is unitary and $D=\operatorname{diag}\left(m_{e}, m_{e}, m_{\tau}\right)$ (recall that we choose $m_{e}=m_{\mu}$ ). Clearly, $U$ can be taken to have unit determinant in general. The final step is to apply a Cartan decomposition [4] on $U$. The involution of choice here will allow us to break $U$ into the product of a matrix in $U(2) \times U(1)$ and a matrix generated by the remaining generators of $S U(3)$. At this point, to illustrate the general procedure, we explicitly perform steps outlined in the Appendix. The Cartan decomposition theorem (see the Appendix for a statement of the theorem and more details) then allows us to write

$$
U=k \exp (\mathbf{p})
$$

where $k \in U(2) \times U(1)$ and $\mathbf{p}=\sum_{j=4}^{7} i a_{j} \lambda_{j} / 2, a_{j}$ are real numbers and $\lambda_{i}$ are the Gell-Mann matrices. Note that $\mathbf{p}$ is described by 4 parameters, the $a_{j}$. The final form of the matrix $R$ 
is then

$$
\begin{aligned}
R & =\exp (-\mathbf{p})\left(\begin{array}{cc}
U_{2 \times 2}^{\dagger} & 0 \\
0 & e^{-i \alpha}
\end{array}\right)\left(\begin{array}{ccc}
m_{e} & 0 & 0 \\
0 & m_{e} & 0 \\
0 & 0 & m_{\tau}
\end{array}\right)\left(\begin{array}{cc}
U_{2 \times 2} & 0 \\
0 & e^{i \alpha}
\end{array}\right) \exp (\mathbf{p}) \\
& =\exp (-\mathbf{p})\left(\begin{array}{ccc}
m_{e} & 0 & 0 \\
0 & m_{e} & 0 \\
0 & 0 & m_{\tau}
\end{array}\right) \exp (\mathbf{p}) .
\end{aligned}
$$

The main result following from (11) is that it only 6 parameters are required to describe the matrix $R$ in this way. They are the two eigenvalues and the $4 a_{j}$. This is in contrast to the usual 9 for a general $3 \times 3$ Hermitian matrix.

The decomposition of $R$ given by (11) demonstrates the fact that the value of $N_{\text {general }}$ is reduced when symmetries are imposed. In this case, the usual 18 is decreased to 15 , of which 7 are real parameters and 8 are phases. As a check, the symmetry breaking pattern is

$$
U(3)_{L} \times U(3)_{E} \rightarrow U(2)_{e} \times U(1)_{\tau}
$$

Thus, there are 13 broken symmetry generators, $N_{\text {broken }}=13$. Using the fact that $N_{\text {general }}=$ 15 and using Eq. (2) we get

$$
N_{\text {phys }}=N_{\text {general }}-N_{\text {broken }}=15-13=2 .
$$

Indeed there are two flavor parameters in this model, $m_{e}$ and $m_{\tau}$.

Now consider a more general model with one term of the form in (7), $Y_{i j} \phi_{i}^{(1)} \phi_{j}^{(2)}$. Without any restrictions, $2 n^{2}$ parameters would be required to describe $Y$ in a general basis. Whenever symmetries are imposed on such terms, this number is reduced. The degeneracies of the matrix eigenvalues ensure that one can always parametrize the matrix with fewer parameters than one would naïvely expect. As a first step in proving the general formula, consider imposing an $n_{1}$-fold eigenvalue degeneracy on $Y$, with $1<n_{1} \leq n$. Since $Y$ can be diagonalized, this is equivalent to imposing an $S U\left(n_{1}\right)$ symmetry. Using results obtained in the Appendix, the required number of parameters is reduced by $n_{1}^{2}-1$ and thus $N_{\text {general }}=2 n^{2}-n_{1}^{2}+1$ out of which, $n^{2}+1-n_{1}\left(n_{1}+1\right) / 2$ are real and $n^{2}-n_{1}\left(n_{1}-1\right) / 2$ are phases.

With this result for an imposed $S U\left(n_{1}\right)$ symmetry, it is possible to iteratively extend the symmetry group to $S U\left(n_{1}\right) \times \cdots \times S U\left(n_{k}\right)$. For each imposed $S U\left(n_{j}\right), n_{j}^{2}-1$ parameters can be removed. Thus, the most general result for an $n$ general model with two $n$ fields 
transforming in the (anti-)fundamental of the imposed symmetry group is

$$
N_{\text {phys }}=2 n^{2}-\sum_{j=1}^{k}\left(n_{j}^{2}-1\right) .
$$

In terms of real parameters, $N_{\text {phys }}^{(r)}$, and phases, $N_{\text {phys }}^{(i)}$, the result is

$$
N_{\text {phys }}^{(r)}=n^{2}-\sum_{j=1}^{k}\left(\frac{n_{j}\left(n_{j}+1\right)}{2}-1\right), \quad N_{\text {phys }}^{(i)}=n^{2}-\sum_{j=1}^{k} \frac{n_{j}\left(n_{j}-1\right)}{2} .
$$

Some complications arise when more terms are added to the potential, particularly when one field appears in multiple potential terms. The cases of a full model symmetry and a sector symmetry cease to be the same as the interaction matrices cannot always be diagonalized concurrently with the gauge interactions. The case of a sector symmetry is trivial to extend. In this case, the symmetry must hold if the interaction matrices in the sector were diagonalized. In this diagonal basis, a certain number of eigenvalues need to be degenerate in order for the symmetry to be manifest. The case of an interaction matrix with degenerate eigenvalues was discussed above and applies also to this case. In particular, the rule (15) apply to each individual interaction matrix. For model-wide symmetries, there are correlations between the change of basis matrices allowed in different terms. We demonstrate a general procedure for determining the correlations below.

Consider a model with three fields $\phi^{(k)}$ that have $n$ generations each. Suppose further that the non-gauge interaction terms have the form

$$
L=Y_{i j}^{(2)} \phi_{i}^{(1)} \phi_{j}^{(2)} \cdots+Y_{i j}^{(3)} \phi_{i}^{(1)} \phi_{j}^{(3)} \cdots
$$

A typical example of a part of a model with interactions of this form is the quark-sector Yukawa interactions in the standard model. An $S U\left(n_{1}\right) \times \cdots \times S U\left(n_{k}\right)$ symmetry is imposed with all fields having their first $n_{1}+\cdots+n_{k}$ components transform in the fundamental. Naïvely, one might expect the number of parameters to be simply twice that of the oneinteraction-term model with the same symmetry. However, there is a reduction in the number of parameters due to the fact that the change of basis matrix $U$ in (9) must be the same for both Yukawa matrices in order for the symmetry to hold in some basis. Of the physical parameters subtracted off in (15), $\sum_{j}\left(n_{j}-1\right)$ were real eigenvalues that are now degenerate and $\sum_{j} n_{j}\left(n_{j}-1\right) / 2$ real parameters and phases were parameters in $U$. Thus, since $n$ of the phases of $U$ always multiply out independent of the symmetry, the $U$ matrix has the same number of real parameters and phases

$$
\frac{n(n-1)}{2}-\sum_{j=1}^{k} \frac{n_{j}\left(n_{j}-1\right)}{2} .
$$


Thus, we count twice the number of parameters as in the one term case, then subtract off the number of parameters in each repeated $U$ matrix. Using this counting, we find that the total number of parameters required is

$$
N_{\text {general }}^{(r)}=\frac{n(3 n+1)}{2}-\sum_{j=1}^{k} \frac{\left(n_{j}+4\right)\left(n_{j}-1\right)}{2}, \quad N_{\text {general }}^{(i)}=\frac{n(3 n+1)}{2}-\sum_{j=1}^{k} \frac{n_{j}\left(n_{j}-1\right)}{2} .
$$

If the symmetry is only required to hold in the Yukawa sector, but may be broken by the weak interactions, then there really are twice as many parameters in this case as in the case with one interaction term. That is

$$
N_{\text {general }}^{(r)}=2 n^{2}-2 \sum_{j=1}^{k}\left(\frac{n_{j}\left(n_{j}+1\right)}{2}-1\right), \quad N_{\text {general }}^{(i)}=2 n^{2}-2 \sum_{j=1}^{k} \frac{n_{j}\left(n_{j}-1\right)}{2} .
$$

Finally, if we demand only that the first term has such a symmetry, but allow the symmetry to be broken by the other term, then only $Y^{(2)}$ is restricted. In a general basis, we subtract off the parameters of $U$ that are unnecessary for that matrix

$$
N_{\text {general }}^{(r)}=2 n^{2}-\sum_{j=1}^{k}\left(\frac{n_{j}\left(n_{j}+1\right)}{2}-1\right), \quad N_{\text {general }}^{(i)}=2 n^{2}-\sum_{j=1}^{k} \frac{n_{j}\left(n_{j}-1\right)}{2} .
$$

Any other model can be handled by accounting for the appropriate relation among the $U$ matrices, described in one of the cases (18), (19) or (20).

\section{PARAMETER COUNTING IN THE MSSM}

Even with imposed R-parity, the MSSM has 124 parameters, which is much more than the 19 of the standard model [5, 6]. In order to make any specific, quantitative predictions using the model, it is necessary to make some assumptions about the flavor structure of the model. One of the most popular models that does so is the constrained MSSM (cMSSM), which has only 4 new parameters and one undetermined sign. The cMSSM involves a number of arbitrary assumptions about the parameters that appear in the low-energy Lagrangian. A different approach is to start imposing symmetries on the interactions at some UV scale, which we can then run down to the scales being studied. In order to see how this approach works and how the rules derived in section [I help us in studying the MSSM, we consider a toy version of the MSSM.

The toy model has only two generations of quarks, no leptons and exact R-parity. The superpotential for quark multiplets is

$$
W=Y_{i j}^{U} Q_{i} U_{j} H_{u}+Y_{i j}^{D} Q_{i} D_{j} H_{d},
$$


where $Y_{i j}^{Q}$ are $2 \times 2$ complex matrices. See for example [6] for the choice of conventions for representations under the MSSM gauge group. The SUSY-breaking potential for the squarks is given by

$$
V_{\mathrm{soft}}=\left(A_{i j}^{U} \tilde{Q}_{i} \tilde{U}_{j} H_{u}+A_{i j}^{D} \tilde{Q}_{i} \tilde{D}_{j} H_{d}+\text { h.c. }\right)+\left(M^{2}\right)_{i j}^{Q} \tilde{Q}_{i}^{\dagger} \tilde{Q}_{j}+\left(M^{2}\right)_{i j}^{U} \tilde{U}_{i}^{\dagger} \tilde{U}_{j}+\left(M^{2}\right)_{i j}^{D} \tilde{D}_{i}^{\dagger} \tilde{D}_{j}
$$

where $A_{i j}^{Q}$ are complex $2 \times 2$ matrices, and $\left(M^{2}\right)_{i j}^{F}$ are Hermitian $2 \times 2$ matrices.

Before restricting the model, we compute the number of flavor parameters in this toy MSSM. There are four $2 \times 2$ complex matrices and three $2 \times 2$ Hermitian matrices, which in the absence of symmetries gives the counting

$$
N_{\text {general }}^{(r)}=25, \quad N_{\text {general }}^{(i)}=19 .
$$

The full $U(2)^{3}$ flavor symmetry is broken by the interaction terms

$$
U(2)^{3} \rightarrow U(1)_{B}
$$

Using (2), we then find that

$$
N_{\text {phys }}^{(r)}=22, \quad N_{\text {phys }}^{(i)}=11 .
$$

The non-supersymmetric model with the same gauge and matter content has only 5 real parameters in the quark sector.

As in the non-supersymmetric case, there are a number of ways to impose a symmetry. Obviously, we could require the symmetry to hold through all sectors of the model. However, the symmetry could also be imposed on the SUSY-breaking sector and broken by the SUSY sectors. It could be imposed on the two potentials, but broken by weak interactions. Finally, it could be imposed on the up quarks only, but broken by the down quarks or vice versa.

Consider the various ways of imposing a $U(1)$ symmetry on the lighter generation of quarks. This symmetry will automatically guarantee a second $U(1)$ for the heavy quarks. The least restrictive ways to impose the symmetry are to demand either that it hold only for the up quarks or only in the soft SUSY-breaking potential. It turns out that both scenarios have the same number of parameters. In the case where symmetry is imposed only on the up quark matrices, the only restriction is that all the up quark interaction matrices be simultaneously diagonalizable. If the matrices are written in the form (9), then all their $U$ and $\Phi$ matrices must be the same up to an overall diagonal phase matrix. The down interaction matrices are not affected by this restriction. If the symmetry is imposed for both types of quarks, but only in $V_{\text {soft }}$, then all the $U$ matrices must be the same within the SUSY-breaking sector. Both cases lead to the counting:

$$
N_{\text {general }}^{(r)}=21, \quad N_{\text {general }}^{(i)}=15 .
$$


Since the imposed symmetry is broken by other sectors, the symmetry breaking is

$$
U(2)^{3} \rightarrow U(1)_{B}
$$

With (2), it is then easy to see that the number of physical parameters is given by

$$
N_{\text {phys }}^{(r)}=18, \quad N_{\text {phys }}^{(i)}=7 .
$$

The number of parameters is further reduced if we demand that the symmetry hold for both potentials and for both types of quarks. Not only are the $U$ matrices now correlated, but so are the $\Phi$ matrices. The number of parameters in a general basis is

$$
N_{\text {general }}^{(r)}=18, \quad N_{\text {general }}^{(i)}=12 .
$$

There is no additional symmetry for the full model, and we count that

$$
N_{\text {phys }}^{(r)}=15, \quad N_{\text {phys }}^{(i)}=4 .
$$

The next more restrictive case is imposing the $U(1)$ throughout the model. Progressing to this case is as simple as extending the correlations from the previous cases to the entire model, so that

$$
N_{\text {general }}^{(r)}=17, \quad N_{\text {general }}^{(i)}=11 .
$$

The extra $U(1)$ symmetry now holds on the model so part of the flavor symmetry is restored

$$
U(2)^{3} \rightarrow U(1)_{u} \times U(1)_{c} .
$$

Thus, the number of physical parameters is given by

$$
N_{\text {phys }}^{(r)}=14, \quad N_{\text {phys }}^{(i)}=4 .
$$

Next, we study models where we impose minimal flavor violation (MFV) on the Yukawas and their supersymmetry-breaking extensions. MFV is defined in the spurion formalism by saying that the only flavor-violating spurions are the standard model Yukawa matrices. To leading order, this forces $A^{F}=a^{F} Y^{F}$, where $a^{F}$ is a complex number, and $\left(M^{2}\right)^{F}=\left(m^{2}\right)^{F} 1$, where $\left(m^{2}\right)^{F}$ is a real number. The parameter counting in the SUSY-breaking sector is as follows. There are two additional parameters for each three-scalar coupling and one extra for each mass. In the end, we find that in a general basis

$$
N_{\text {general }}^{(r)}=13, \quad N_{\text {general }}^{(i)}=10 .
$$

Only baryon number is left after breaking the symmetry, so that

$$
N_{\text {phys }}^{(r)}=10, \quad N_{\text {phys }}^{(i)}=2 .
$$


The cMSSM is a restriction of the MFV case. It is assumed that at some high scale all the scalar masses are equal, all the three-scalar couplings $a^{F}$ are equal and all the gaugino masses are equal. In the full model with leptons, these restrictions hold between baryons and leptons as well. Furthermore, the new interactions are assumed to be CP-conserving so that there are no new $\mathrm{CP}$ violating physical phases. With these conditions, the counting in a general basis is

$$
N_{\text {general }}^{(r)}=10, \quad N_{\text {general }}^{(i)}=8,
$$

so that in the physical basis

$$
N_{\text {phys }}^{(r)}=7, \quad N_{\text {phys }}^{(i)}=0 .
$$

The non-supersymmetric analogue of this model had only 5 flavor parameters: four quark masses and a mixing angle. Thus, there are two new flavor parameters here which we can take to be the SUSY-breaking squark mass $m_{0}^{2}$ and the triscalar coupling $a_{0}$. These two additional parameters in the quark sector, together with the SUSY-breaking Higgs parameter $b$ and the gaugino mass $m_{1 / 2}$, are the only new parameters. The superpotential mass parameter $\mu$ can be related to the Higgs VEV and is not counted as new. An extra undetermined sign comes from moving to a more convenient parametrization where the Higgs parameters $\mu$ and $b$ are traded for $m_{Z}$ and $\tan \beta$. The two sets of parameters contain the same information up to the sign of $\mu$ which is not fixed by fixing $m_{Z}$ and $\tan \beta$. This ambiguity arises from the fact that the scalar Higgs potential of the MSSM depends only on $|\mu|^{2}$ and not on $\mu$.

Most of the counting outlined in (23)-(37) above extends trivially to constraining the full MSSM. The main complication is the additional generation in the fermion sectors. The additional generation allows an $S U(2)$ flavor symmetry to be imposed. This type of symmetry can then be handled using the rules derived in section II]. Maintaining exact R-parity, the superpotential for the fermion multiplets is given by

$$
W=Y_{i j}^{U} Q_{i} U_{j} H_{u}+Y_{i j}^{D} Q_{i} D_{j} H_{d}+Y_{i j}^{L} L_{i} E_{j} H_{d},
$$

where $Y_{i j}^{F}$ are $3 \times 3$ complex matrices and $\mu$ is a complex number. The SUSY-breaking potential for the fields in these multiplets is given by:

$$
\begin{aligned}
& V_{\text {soft }}=\left(A_{i j}^{U} \tilde{Q}_{i} \tilde{U}_{j} H_{u}+A_{i j}^{D} \tilde{Q}_{i} \tilde{D}_{j} H_{d}+A_{i j}^{L} \tilde{L}_{i} \tilde{E}_{j} H_{d}+\text { c.c. }\right)+ \\
& \quad\left(M^{2}\right)_{i j}^{Q} \tilde{Q}_{i}^{\dagger} \tilde{Q}_{j}+\left(M^{2}\right)_{i j}^{U} \tilde{U}_{i}^{\dagger} \tilde{U}_{j}+\left(M^{2}\right)_{i j}^{D} \tilde{D}_{i}^{\dagger} \tilde{D}_{j}+\left(M^{2}\right)_{i j}^{L} \tilde{L}_{i}^{\dagger} \tilde{L}_{j}+\left(M^{2}\right)_{i j}^{E} \tilde{E}_{i}^{\dagger} \tilde{E}_{j},
\end{aligned}
$$

where $A_{i j}^{F}$ are complex $3 \times 3$ matrices and $\left(M^{2}\right)_{i j}^{F}$ are Hermitian $3 \times 3$ matrices.

The results of the parameter counting for various imposed symmetries are described in table II. The four columns show the number of real and imaginary parameters in a general basis and in the physical basis for the potential of the chiral flavored fields. The first row 


\begin{tabular}{cccccc}
\hline Imposed Symmetry & Broken By & $N_{\text {general }}^{(r)}$ & $N_{\text {general }}^{(i)}$ & $N_{\text {phys }}^{(r)}$ & $N_{\text {phys }}^{(i)}$ \\
\hline None & 84 & 69 & 69 & 41 \\
Fermion Family & SUSY Interaction & 66 & 51 & 51 & 23 \\
& Weak Interactions & 51 & 36 & 36 & 10 \\
& All & 48 & 33 & 33 & 9 \\
SU (2) Flavor & SUSY Interaction & 56 & 49 & 41 & 21 \\
& Weak Interactions & 37 & 30 & 23 & 6 \\
& All & 35 & 28 & 22 & 6 \\
Leading MFV & & 35 & 30 & 20 & 4 \\
$S U(3)$ Flavor & & 20 & 21 & 11 & 3 \\
\hline
\end{tabular}

TABLE I: Parameter counting in the chiral multiplet potentials of the MSSM with various imposed symmetries in the potentials only and in the entire Lagrangian for the model. The large $S U(N)$ symmetries are necessarily broken, possibly spontaneously [7].

gives the counting for the case when no symmetry is imposed. This is the MSSM-124 model. The second through fourth lines describe the case where only $U(1)$ family symmetry is imposed. On the second line, the symmetry is broken by the superpotential. On the third line, it is broken by weak gauge interactions. On the fourth line, it holds through all renormalizable terms in the model. The fifth through seventh lines describe the case where $S U(2)$ is imposed with the first two generations transforming as a doublet and the third as a singlet. The same three symmetry-breaking possibilities are presented. On the eighth line, we present the case of MFV where only the leading term in powers of the Yukawa matrices is kept. Finally, the case with maximal $S U(3)$ flavor symmetry is presented.

\section{CONCLUSIONS}

It is clear that the Standard Model is a low energy description of a more fundumental theory. The introduction of new states and symmetries into the Lagrangian adds many new interaction matrices. The hierarchy of the SM Yukawa matrices as well as the new physics flavor puzzle [2] motivate the idea that new flavor symmetries or approximate symmetries could exist in more fundamental interactions. If such symmetries exist, then parameter counting may be non-trivial. The number of parameters required in a general basis is less 
than if the symmetries were not imposed. We derived rules for accounting for this reduction in the number of parameters. We demonstrated the analysis for a series of toy models, leading up to counting the number of parameters in the MSSM with various imposed flavor symmetries. The results obtained for the MSSM are summarized in Table I. However, the methods used above are general and can be used to study other potential UV completions of the Standard Model.

\section{Acknowledgments}

We thank Yossi Nir and João Silva for helpful discussions. This research is supported by the NSF grant PHY-0355005.

\section{APPENDIX A: CARTAN DECOMPOSITION OF A UNITARY MATRIX}

The Cartan decomposition theorem is a theorem about semisimple Lie groups that gives a decomposition for elements of the group. In all the cases that we consider, the matrix we would like to decompose is an element of the semisimple Lie group $U(n)$. It is trivial to factor out the overall phase of such a matrix, and thus we consider below the decomposition of a matrix $U \in S U(n)$ for simplicity.

The mathematical definitions and theorems can be found, for example, in [4]. The specific decomposition process is inspired by the work of [8]. In order to understand the idea of a Cartan decomposition, we need to make some definitions.

Definition 1. Let $\mathfrak{g}$ be a semisimple Lie algebra. An automorphism $\theta$ of $\mathfrak{g}$ with square equal to the identity is called an involution. An involution is a Cartan involution if the symmetric bilinear form

$$
B_{\theta}(X, Y)=-B(X, \theta Y)
$$

is positive definite, where $B$ is the Killing form of $\mathfrak{g}$.

The second definition is slightly technical, but for practical purposes, the involutions we use satisfy this condition. For more details, please see [4]. Since $\theta^{2}=1, \theta$ has eigenvalues \pm 1 on $\mathfrak{g}$. Thus, we can decompose $\mathfrak{g}=\mathfrak{l} \oplus \mathfrak{p}$, where $\mathfrak{l}$ and $\mathfrak{p}$ are the eigenspaces of $\theta$ corresponding to eigenvalues +1 and -1 respectively. This is the Cartan decomposition on a Lie algebra level. It is trivial to see by applying the involution that $[\mathfrak{l}, \mathfrak{l}]=\mathfrak{l}$; that is, the commutator of any two Lie algebra elements with eigenvalue 1 under $\theta$ has eigenvalue 1 . This result means that the eigenspace $\mathfrak{l}$ is actually a Lie subalgebra. Extending this to the Lie group level is non-trivial and the theorem is due to Cartan. 
Theorem 1. Let $G$ be a semisimple Lie group with Lie algebra $\mathfrak{g}$. Let $\theta$ be a Cartan involution on $\mathfrak{g}$. Let $\mathfrak{g}=\mathfrak{l} \oplus \mathfrak{p}$ be the eigenspace decomposition for $\theta$. Finally, let $K$ be the subgroup of $G$ with Lie algebra $\mathfrak{l}$. Then

1. there exists a Lie group automorphism $\Theta$ of $G$ with differential $\theta$ and with $\Theta^{2}=1$,

2. the subgroup of $G$ that is invariant under $\Theta$ is $K$,

3. the mapping $K \times \mathfrak{p} \rightarrow G$ given by $(k, \mathbf{p}) \mapsto k \exp (i \mathbf{p})$ is a diffeomorphism.

The first consequence can be interpreted as saying that for group elements infinitesimally different from the identity, the relation $\Theta(g)=1+i \epsilon \theta(\mathbf{g})+O\left(\epsilon^{2}\right)$ holds. For any group element, this can be extended to $\Theta(g)=\exp (i \theta(g))$. The third consequence is the main result that we need in order to perform the decomposition. Effectively, it allows us to factor an element $g \in G$ into a product of an element $k \in K$ and another element of $S U(n)$ given by $\exp (i \mathbf{p})$ for $\mathbf{p} \in \mathfrak{p}$ by using the fact that the map defined in condition 3 is a diffeomorphism and that $\mathfrak{g}=\mathfrak{k} \oplus \mathfrak{p}$.

Now, consider the group $G=S U(n)$. Suppose we want to factor an element $g \in G$ into a product of an element $k$ which is block diagonal with the first $n_{1} \times n_{1}$ block an element of $S U\left(n_{1}\right)$ for $n_{1}<n$ and another element $p \in S U(n)$ whose generators are all different from those of $k$. Along similar lines to [8], we choose an involution

$$
\theta(\mathbf{g})=\left(\begin{array}{cc}
-1_{n_{1}} & 0 \\
0 & 1_{n-n_{1}}
\end{array}\right) \mathrm{g}\left(\begin{array}{cc}
-1_{n_{1}} & 0 \\
0 & 1_{n-n_{1}}
\end{array}\right)
$$

This involution is in fact a Cartan involution. Furthermore, its eigenspace with eigenvalue +1 is all special unitary matrices that are block diagonal with blocks of size $n_{1} \times n_{1}$ and $\left(n-n_{1}\right) \times$ $\left(n-n_{1}\right)$. This subalgebra is generated by matrices whose upper-left block are generators of $S U\left(n_{1}\right)$ and remaining entries are zero, whose lower-right block are generators of $S U\left(n-n_{1}\right)$ and remaining entries zero, or which are diagonal phase matrices with determinant 1 . The orthogonal eigenspace is generated by the generators whose entries are all off the diagonal block. By the Cartan decomposition theorem, we can then write any $S U(n)$ matrix in the form

$$
U=\left(\begin{array}{cc}
U_{n_{1}} & 0 \\
0 & U_{n-n_{1}}
\end{array}\right) \exp (i \mathbf{p})
$$

where $U_{k}$ is a matrix in $U\left(n_{k}\right)$ with $\operatorname{det} U_{n_{1}} \operatorname{det} U_{n-n_{1}}=1$ and where $\mathbf{p}$ is in the Lie algebra of $S U(n)$ and is generated by matrices whose diagonal blocks are zero. 
Note that this process can be iterated: we can then decompose $U_{n-n_{1}}$ in a similar way. Ultimately, the matrix $U$ can be written in the form

$$
U=\left(\begin{array}{cccc}
U_{n_{1}} & & & \\
& U_{n_{2}} & & \\
& & \ddots & \\
& & & U_{n_{k+1}}
\end{array}\right) \exp (i \mathbf{p})
$$

where $n_{1}+n_{2}+\cdots+n_{k+1}=n, \operatorname{det}\left(U\left(n_{1}\right) U\left(n_{2}\right) \ldots U\left(n_{k}\right)\right)=1$, and $\mathbf{p}$ is a linear combination of generators whose entries are all off the diagonal block. The condition on the determinants can be removed by allowing $U \in U(n)$ rather than $S U(n)$.

The essential result for this work is that a Hermitian matrix with degenerate eigenvalues can be written in terms of fewer parameters than if no degeneracy were present. Let $R$ be a Hermitian matrix that has $k$ degenerate eigenvalues, with the first one, $r_{n_{1}}$, being $n_{1}$-fold degenerate, the second one, $r_{n_{2}}$, being $n_{2}$-fold degenerate, and so on. By spectral decomposition, the matrix can be written as

$$
R=U^{\dagger} D U
$$

where $D$ is diagonal. The matrices $U$ are unitary since $R$ is Hermitian. Now, decompose $U$ using (A4). The decomposition yields

$$
R=\exp (-i \mathbf{p}) U^{\dagger} D U \exp (i \mathbf{p})=\exp (-i \mathbf{p}) D \exp (i \mathbf{p})
$$

such that

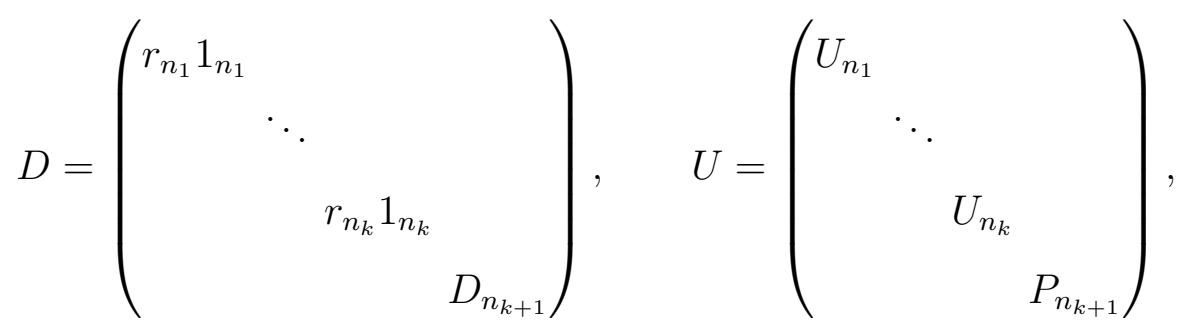

where $D_{n_{k+1}}$ is diagonal matrix and $P_{n_{k+1}}$ is a diagonal matrix of (different) phases. In order to count the number of parameters necessary to describe this matrix, we can count the number of parameters in $U$ before performing the reduction of (A6) and subtract off the number of parameters removed by decomposing. $U$ is an $n \times n$ unitary matrix, which naïvely has $n(n-1) / 2$ real parameters and $n(n+1) / 2$ phases. By decomposition, we removed $\sum_{j} n_{j}\left(n_{j}-1\right) / 2$ real parameters and $\sum_{j} n_{j}\left(n_{j}+1\right) / 2+n-\sum_{j} n_{j}$ phases. In the counting of the phases, the first sum comes from adding up the parameters in the unitary matrices $U_{n_{j}}$ and the second two terms come from adding up the phases in $P_{n_{k+1}}$. Furthermore, the 
number of real parameters in $D$ is $n-\sum_{j}\left(n_{j}-1\right)$. Thus, the number of real parameters in $R$ is

$$
N_{R}^{(r)}=\frac{n(n+1)}{2}-\sum_{j}\left(\frac{n_{j}\left(n_{j}+1\right)}{2}-1\right)
$$

and the number of phases in $R$ is

$$
N_{R}^{(i)}=\frac{n(n-1)}{2}-\sum_{j}\left(\frac{n_{j}\left(n_{j}-1\right)}{2}\right) .
$$

[1] A. Santamaria, Phys. Lett. B 305, 90 (1993) arXiv:hep-ph/9302301.

[2] Y. Nir, arXiv:0708.1872].

[3] C. Amsler et al., Physics Letters B667, 1 (2008) http://pdg.lbl.gov.

[4] A. W. Knapp, Lie Groups Beyond an Introduction, Birkhäuser Boston (1996).

[5] S. Dimopoulos, D. Sutter, Nucl. Phys. B452, 496-512 (1995)

[6] H. E. Haber, Nucl. Phys. Proc. Suppl. 101, 217-236 (2001) arxiv:hep-ph/0103095.

[7] R. Barbieri, L. J. Hall, S. Raby, A. Romanino, Nucl. Phys. B493, 3-26 (1997) arxiv:hep-ph/9610449

[8] P. P. Divakaran, R. Ramachandran, Pramana 14, 47-56 (1980). 